\title{
Dynamic Contrast for Plant Phenotyping
}

\author{
Zsolt Kelemen," Ruikang Zhang," Lionel Gissot, Raja Chouket, Yannick Bellec, Vincent Croquette, \\ Ludovic Jullien,* Jean-Denis Faure,* and Thomas Le Saux*
}

Cite This: ACS Omega 2020, 5, 15105-15114

Read Online

ACCESS | Lill Metrics \& More | 回 Article Recommendations ｜（s Supporting Information

ABSTRACT: Noninvasiveness, minimal handling, and immediate response are favorable features of fluorescence readout for highthroughput phenotyping of labeled plants.Yet, remote fluorescence imaging may suffer from an autofluorescent background and artificial or natural ambient light. In this work, the latter limitations are overcome by adopting reversibly photoswitchable fluorescent proteins (RSFPs) as labels and Speed OPIOM (out-of-phase imaging after optical modulation), a fluorescence imaging protocol exploiting dynamic contrast. Speed OPIOM can efficiently distinguish the RSFP signal from autofluorescence and other spectrally interfering fluorescent reporters like GFP. It can quantitatively assess gene expressions, even when they are weak.
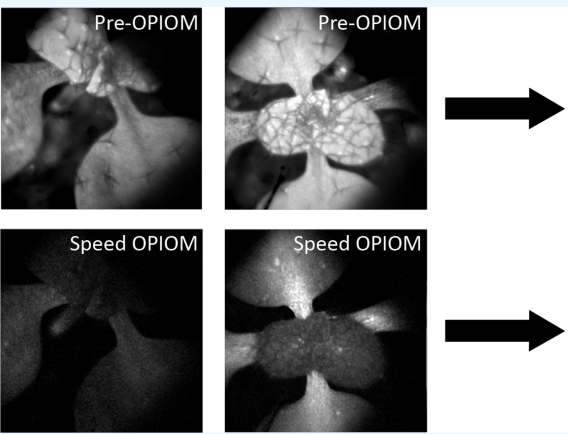

STRESS

DETECTION

It is as quantitative, sensitive, and robust in dark and bright light conditions. Eventually, it can be used to nondestructively record abiotic stress responses like water or iron limitations in real time at the level of individual plants and even of specific organs. Such Speed OPIOM validation could find numerous applications to identify plant lines in selection programs, design plants as environmental sensors, or ecologically monitor transgenic plants in the environment.

\section{INTRODUCTION}

The rise of the human population combined with the requirement for a sustainable agriculture requires developing modern techniques for plant breeding, cultivation, and management of crops. Thus, high-throughput phenotyping tools providing quantitative analyses of plant structure and function are actively sought for developing highly productive strains or monitoring plants to determine their physiological state. $^{1-3}$ A fluorescence readout is herein advantageous: it is not invasive, requires minimal handling, and provides an immediate response.

Endogenous fluorescence emission can be analyzed down to individual plants through the canopy, up to the field or even regional scales. $^{4-7}$ Since it increases markedly in response to stress, ${ }^{8}$ it has been used to extract physiological information on plants. ${ }^{9,10}$ In addition to investigation of optical properties of plants, fluorescent proteins (FPs $)^{11}$ have been proposed to further expand opportunities for fluorescence reporting upon visualizing physiological processes and gene expression under the control of inducible promoters acting as noninvasive sensors to stress situations. ${ }^{12-16}$ However, imaging of fluorescent labels is challenging in plants, which accumulate a large diversity of endogenous fluorescent compounds, in particular, upon stress conditions. Hence, FP-mediated imaging can suffer from a significant autofluorescent background within a broad range of wavelengths (from UV to IR). Second, the observation of FP fluorescence, as well as endogenous fluorescence, requires either a low-light environ- ment or light-proof devices, which limits its use for live recording of plants. The possibility to selectively record fluorescence emission with minimal contribution from an autofluorescence background and artificial or natural ambient light would definitively open new avenues for plant phenotyping.

A fluorescence signal can be discriminated against ambient light by tailoring the excitation light. To subtract images recorded under ambient lighting with and without excitation light is a first approach. ${ }^{17}$ However, subtraction suffers from a temporally varying lighting environment and it cannot be used if ambient light is too strong as a consequence of the shot noise. Pulsed excitation light of $\mu \mathrm{s}-\mathrm{ms}$ duration associated with gated detection has provided a second approach for fluorescence imaging under room light to sunlight conditions. ${ }^{9,18-21}$ Eventually, lock-in imaging has been implemented to distinguish a fluorescence signal from ambient light by retrieving the background-free modulated component of fluorescence under periodic modulation of excitation light. ${ }^{22,23}$ In contrast to the two preceding approaches, it benefits from a higher signal-to-noise ratio (SNR) due to frequency-domain

Received: March 3, 2020

Accepted: June 2, 2020

Published: June 16, 2020 

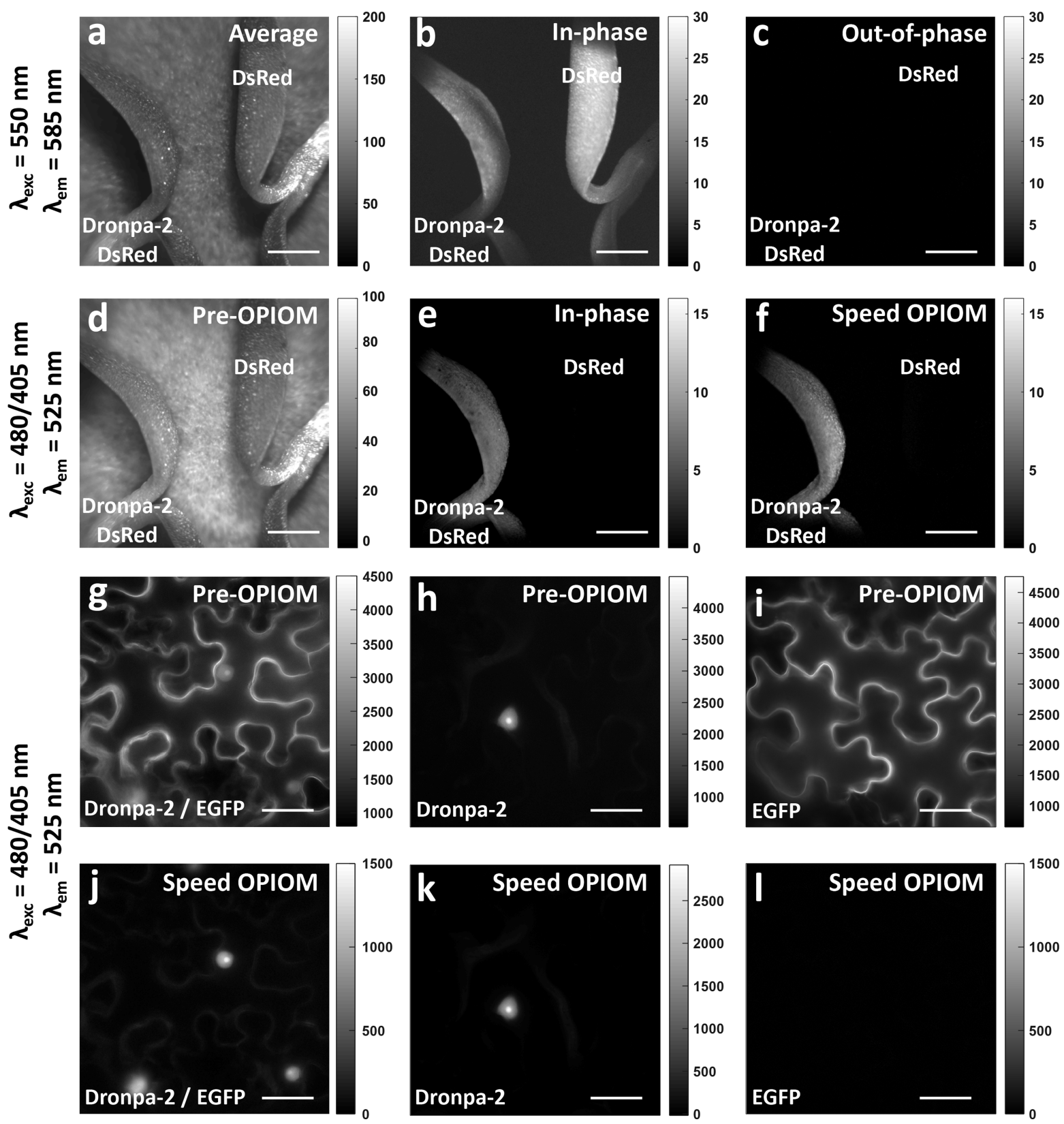

Figure 1. RSFPs as selection markers of low spectral interference. $(a-f)$ Selective imaging of DsRed (a-c: red channel) and Dronpa-2 (d-f: green channel) against autofluorescence and ambient light in C. sativa leaves (left: labeled plant expressing both DsRed and Dronpa-2; right: labeled plant only expressing DsRed as a control). Images were taken under modulated illumination (a-c: $\lambda_{\text {exc }}=550 \mathrm{~nm}$ and $\lambda_{\text {em }}=585 \mathrm{~nm} ; \mathrm{d}-\mathrm{f}: \lambda_{\text {exc, }, 1}=480 \mathrm{~nm}$, $\lambda_{\text {exc }, 2}=405 \mathrm{~nm}$, and $\lambda_{\text {em }}=525 \mathrm{~nm}$ ). Averaged (a, Pre-OPIOM in d), in-phase (b, e), and out-of-phase (c, Speed OPIOM in f) images acquired in an outdoor condition under indirect sunlight. Average images can hardly evidence the presence of DsRed or Dronpa-2 against the background signal (autofluorescence and ambient light). In contrast, Dronpa-2 is unambiguously imaged by Speed OPIOM. Scale bars, $1 \mathrm{~mm}$. (g-1) Selective Speed OPIOM epifluorescence microscopy imaging of Dronpa-2 against EGFP in N. benthamiana leaves. Pre-OPIOM (g-i) and Speed OPIOM (j-1) images of constructs expressing Dronpa-2 and/or EGFP (g, j: PMA4-EGFP and Fbr-Dronpa-2; h, k: Fbr-Dronpa-2; i, l: PMA4-EGFP). PreOPIOM simultaneously imaged both EGFP and Dronpa-2. In contrast, Dronpa-2 was selectively imaged by Speed OPIOM. Scale bars, $50 \mu \mathrm{m}$. Illumination conditions: see Table S1.

noise filtering. In addition, it is insensitive to irregular fluctuation of ambient light. However, none of these approaches leads to selective extraction of the signal of a targeted fluorescent label against autofluorescence. Autofluorescence is often removed in the spectral domain by its handling as a fluorophore and further image processing. ${ }^{24,25}$ However, this approach is limited when autofluorescence arises from diverse substances at specific locations. ${ }^{26}$ Moreover, image processing may be compromised by noise, which may be critical at low illumination.

The preceding limitations can be overcome with dynamic contrast, which relies on light as a perturbation parameter and further signal processing to selectively retrieve the signal from a targeted fluorophore upon eliminating spectral interferences from ambient light, autofluorescence, or other fluorescent labels. ${ }^{27}$ The fluorescence lifetime has been exploited early on to discriminate fluorophores ${ }^{28}$ and it was applied to eliminate 
an autofluorescence background in plants. ${ }^{29}$ To overcome the limitations of a narrow dispersion of the fluorescence lifetimes and facilitate discrimination, dynamic contrast has been more recently proposed for selective imaging of reversibly photoswitchable fluorescent proteins (RSFPs). ${ }^{30,31}$ The latter benefit from a wider range of relaxation times and they have been successfully used for selective imaging in plants. ${ }^{32-34}$ In this account, we exploit the Speed OPIOM (out-of-phase imaging after optical modulation) imaging protocol of dynamic contrast, which we recently implemented on a fluorescence macroscope. ${ }^{3,34}$ Designed to image macroscale samples with minimal preparation, enhanced sensitivity, and excellent signalto-noise ratio originating from lock-in amplification, ${ }^{33,34}$ we here demonstrate that it can be used to image RSFPs in transient as well as in stable plant systems in a context relevant to plant phenotyping. We show that Speed OPIOM can efficiently report on transcriptional activities, both in a qualitative and quantitative manner, and reliably monitor early plant response to biotic stresses, even in the presence of endogenous fluorescence and under ambient light.

\section{RESULTS AND DISCUSSION}

2.1. Speed OPIOM Principle. In OPIOM,,$^{33,35}$ the fluorescent labels are reversibly photoswitchable fluorophores (RSFs), which have been popularized by super-resolution microscopy. Speed OPIOM has specifically exploited RSFPs, which can reversibly photoswitch between two states of distinct brightnesses by using two different wavelengths. In Speed OPIOM, the fluorescence emission is modulated by the periodically modulated illumination involving two light sources at wavelengths $\lambda_{1}$ and $\lambda_{2}$ modulated in the antiphase at angular frequency $\omega$ with respective average light intensities $I_{1}{ }^{0}$ and $I_{2}{ }^{0}$. The Speed OPIOM signal is the amplitude of the quadrature component of the modulated fluorescence emission, which exhibits a resonance in the space of the illumination parameters $\left(I_{2}{ }^{0} / I_{1}{ }^{0}\right.$ and $\left.\omega / I_{1}{ }^{0}\right)$. The Speed OPIOM image of a targeted RSFP is obtained after matching the illumination parameters to its cross sections for fluorescence photoswitching at wavelengths $\lambda_{1}$ and $\lambda_{2}$ using the resonance conditions. In contrast to traditional imaging of simple fluorophores, Speed OPIOM can discard any spectrally interfering contribution from ambient light, not expected to be modulated at angular frequency $\omega$, and autofluorescence, which would only contribute to the amplitude of the in-phase component of the modulated fluorescence emission. ${ }^{33}$ Since the plant tissues are optically complicated, Speed OPIOM application to plant imaging has deserved a specific analysis (see the Supporting Information).

2.2. Speed OPIOM for Selecting Transgenic Plants. Transgenic species are often selected by adding gene coding for a fluorescent protein acting as a reporter to a transfected gene(s) of interest. ${ }^{36}$ However, this approach reduces the accessible wavelength window, which can be exploited for subsequent fluorescence imaging investigations. Speed OPIOM can overcome this limitation since it exploits dynamic contrast and not spectral discrimination to selectively image a fluorescent label.

To illustrate such a situation, we first generated and selected transgenic Camelina sativa plants using DsRed as a selection marker. To image DsRed under ambient light, we first considered using fluorescence imaging at $585 \mathrm{~nm}$ under constant illumination at $550 \mathrm{~nm}$ or similarly to exploit the fluorescence image resulting from averaging the images collected at $585 \mathrm{~nm}$ under an integer number of periods of $550 \mathrm{~nm}$ light modulation. Figure 1a shows that the averaged image can hardly evidence the presence of DsRed against the background signal generated by interference of ambient light (indirect sunlight). Hence, we imaged the in-phase amplitude of the modulated fluorescence emission from the leaves to benefit from lock-in amplification, which eliminates any contribution of light varying at a frequency range differing from the one applied on the light source. As evidenced by the dark background, we could overcome the interference of ambient light (Figure 1b). Eventually, the out-of-phase amplitude of the fluorescence modulation at $585 \mathrm{~nm}$ vanished as expected from the instantaneous response of DsRed fluorescence to any light change (Figure 1c).

We then proceeded in implementing Speed OPIOM to image transgenic $C$. sativa doubly labeled with DsRed and Dronpa-2, ${ }^{37}$ which is an RSFP from the Dronpa family absorbing light and emitting fluorescence at 480 and $525 \mathrm{~nm}$, respectively. ${ }^{38}$ We first generated Pre-OPIOM images under ambient light by averaging the images collected at $525 \mathrm{~nm}$ under an integer number of periods of $480 \mathrm{~nm}$ light modulation (such a protocol is similar to generating images under constant illumination). Both Dronpa-2-labeled and unlabeled plants exhibit comparable signals and poor contrast against the background in the averaged Pre-OPIOM image when light is modulated at Speed OPIOM resonance for Dronpa-2 (Figure 1d). The in-phase image shows a stronger signal in the Dronpa-2-labeled plant than in the unlabeled one (Figure 1e). However, it cannot be used for quantification since both Dronpa-2 and autofluorescence contribute to the inphase image. In contrast, Dronpa-2 is unambiguously imaged in the Dronpa-2-labeled plant in the out-of-phase image of Speed OPIOM, which eliminates the contribution of both ambient light and autofluorescence (Figure 1f).

We eventually evaluated whether Speed OPIOM could be used to select plants with an RSFP reporter in the presence of an FP exhibiting similar excitation/emission properties. We transiently expressed nuclear localized Dronpa-2 (Fbr-Dronpa2) and/or EGFP at the plasma membrane (PMA4-GFP) in leaves of Nicotiana benthamiana. Whereas Pre-OPIOM simultaneously imaged both EGFP and Dronpa- 2 in the cell expressing both FPs (Figure 1g), Dronpa-2 could be selectively imaged with Speed OPIOM (Figure 1j) in line with the control experiments led in cells expressing either Dronpa-2 or EGFP (Figure 1h,i and k,l). Similar results were obtained with leaves labeled with nuclear localized Dronpa-2 and EGFP at the endoplasmic reticulum (PAS1-EGFP) (see Figure S1a-f). Although validated here with Dronpa-2, other RSFPs could be used to overcome the limitation of spectral discrimination. Hence, we eliminated the spectral interference of autofluorescence with four different RSFPs (Dronpa-2, Dronpa-3, rsFastlime, and Padron) in N. benthamiana (see Figure S2). Dronpa has been also expressed in plants $^{32,39}$ and it was previously discriminated from Padron with dynamic contrast in Arabidopsis thaliana. ${ }^{32}$ Since Dronpa-2 allowed us to achieve the fastest acquisitions, it was used in our subsequent observations (see Table S1).

2.3. Speed OPIOM for Quantitative Evaluation of Gene Expression in Transgenic Plants. After validation of Speed OPIOM for qualitatively and robustly evidencing a fluorescent marker during selection of transgenic plants, we evaluated its relevance to quantify gene expression in plant systems with our fluorescence macroscope. We designed three 


\section{$\lambda_{\text {exc }}=480 / 405 \mathrm{~nm}$ $\lambda_{\mathrm{em}}=525 \mathrm{~nm}$}
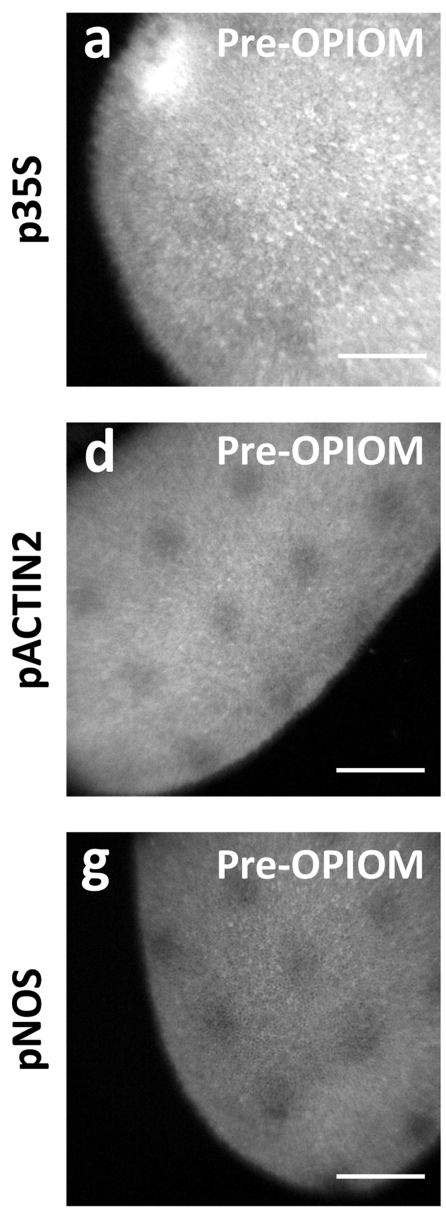
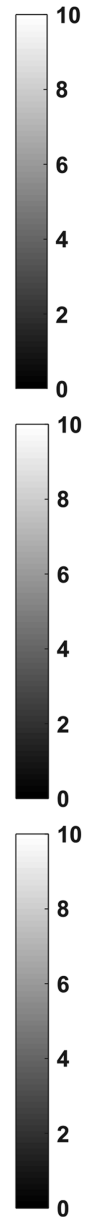
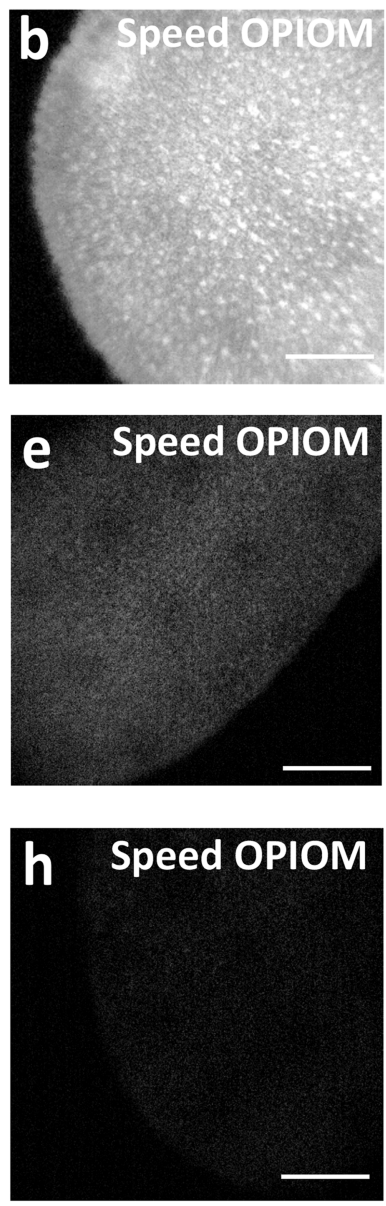

$\lambda_{\text {exc }}=550 \mathrm{~nm}$

$\lambda_{\mathrm{em}}=585 \mathrm{~nm}$
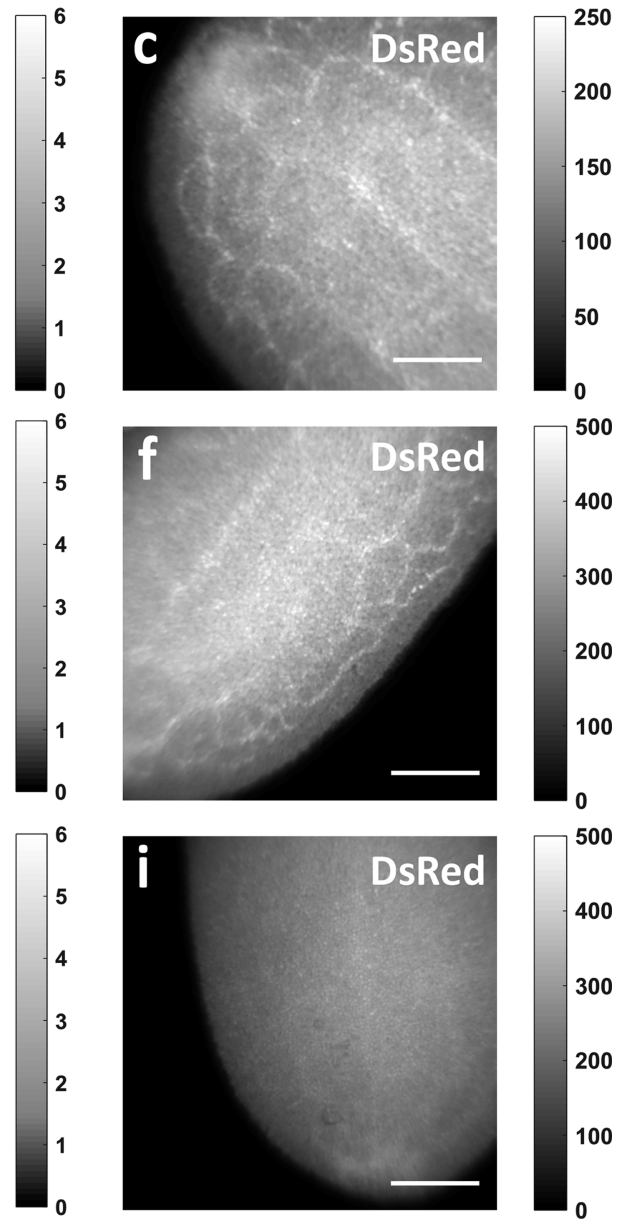

Figure 2. Fluorescence imaging of C. sativa leaves stably expressing Dronpa-2 (imaged with Pre-OPIOM and Speed OPIOM) and DsRed (imaged with in-phase fluorescence imaging). Promoters: p35S (a-c), pACTIN2 (d-f), and pNOS ( $-\mathrm{i})$. Images acquired in the dark. In the Pre-OPIOM images, the signal levels in the green channel are in the same range for all the constructions. In contrast, in the Speed OPIOM images, the signal level is significantly higher for the p35S:Dronpa-2 construct than for the pACTIN:Dronpa-2 and pNOS:Dronpa-2 constructs. Scale bars, 1 mm. Illumination conditions: see Table $S 1$.

constructs in which Dronpa-2 was expressed under the control of promoters of increasing strengths: ${ }^{40}$ pNOS:DRONPA-2, pACTIN2:DRONPA2, and p35S:DRONPA2. In addition, all the constructs carried PCVMV:DsRED as a selection marker.

In the first series of experiments, we analyzed the expression level of Dronpa-2 in leaves of stable transgenic C. sativa plants simultaneously expressing Dronpa-2 (under the control of either pNOS, pACTIN2, or p35S promoter) and DsRed (under the control of the pCVMV promoter). Three independent $\mathrm{T} 1$ lines were selected and Speed OPIOM imaging was subsequently performed in the dark on leaves of independent T2 plants selected for their DsRed fluorescence. To define the most reliable protocol for analyzing the level of transcriptional activity, we analyzed not one but several individual seedlings. Figure 2 displays representative results. Whereas the Dronpa-2 images exhibited a rather homogeneous fluorescence, ${ }^{41,42}$ we noticed that the DsRed image displayed a more heterogeneous fluorescence with the brightest level at the leaf veins. As anticipated from a significant contribution of autofluorescence, the signal levels in the Pre-OPIOM images of the leaves expressing Dronpa-2 under the control of the three distinct promoters were in a same range. In contrast, we noticed that the Dronpa-2 Speed OPIOM signal was significantly higher with the p35S:DRONPA2 construct than with the pACTIN2:DRONPA-2 and pNOS:DRONPA2 ones, which was expected from a drop of promoter strength. Similar results were obtained in $N$. benthamiana leaves transiently expressing Dronpa-2 under the control of the same promoters (see Figure S3).

The preceding data have been quantitatively exploited. In a first step, we analyzed the collected images at the single-pixel level. More specifically, we plotted the intensity of the Speed OPIOM signal as a function of the DsRed one. In leaves expressing Dronpa-2 under the control of the p35S promoter, we observed rather linear correlations suggesting that expression variation observed within the samples (for the same promoters) were most probably caused by general physiological or optical conditions of the expressing cells and not by specific transcriptional regulations (see Figure S4). Furthermore, the slope of the linear fitting was always found close to the ratio of the integrated signals over the whole leaf surface (see Figure S4). In contrast, we obtained poor correlations and much variability for the leaves expressing Dronpa-2 under the control of the pNOS and pACTIN2 

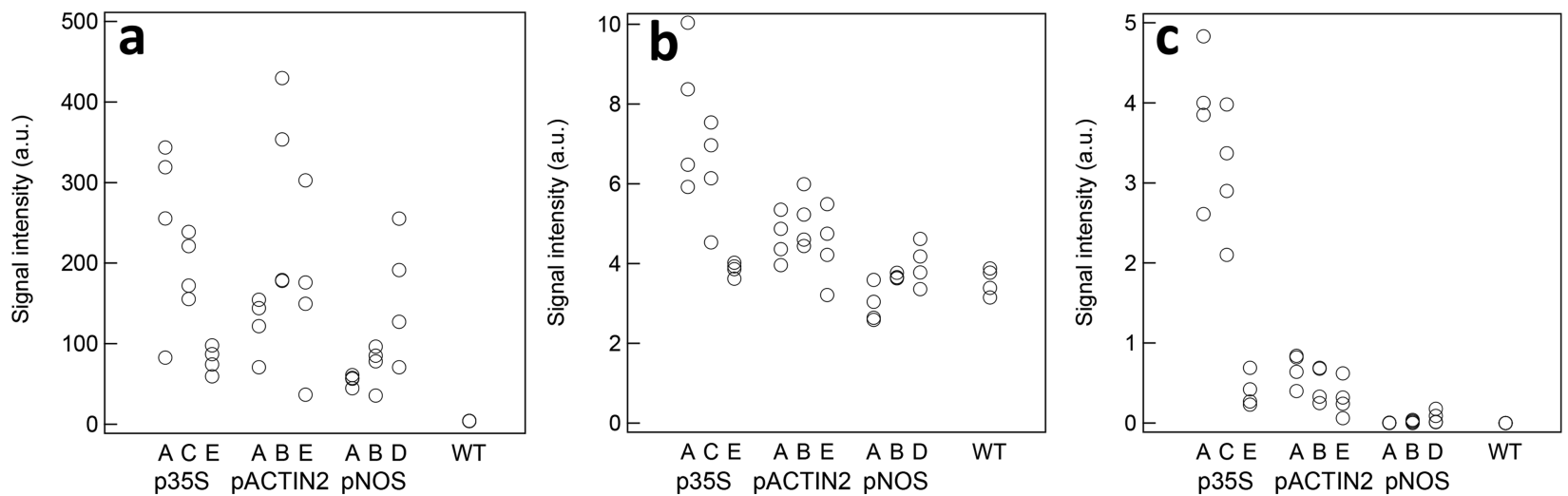

Figure 3. Signals of $C$. sativa seedlings from different imaging channels. In-phase DsRed signal from the images collected at $585 \mathrm{~nm}$ with $550 \mathrm{~nm}$ illumination (a) and Pre-OPIOM (b) and Speed OPIOM (c) signals from the images collected at $525 \mathrm{~nm}$ with 480/405 nm illumination for the three constructs p35S, pACTIN2, and pNOS. Signals have been extracted from integration over the whole surface of the leaf. For each construct, three lines of seedlings ( $\mathrm{ACE}, \mathrm{ABE}$, and $\mathrm{ABD}$ ) have been studied and in each line, four individual samples have been measured.
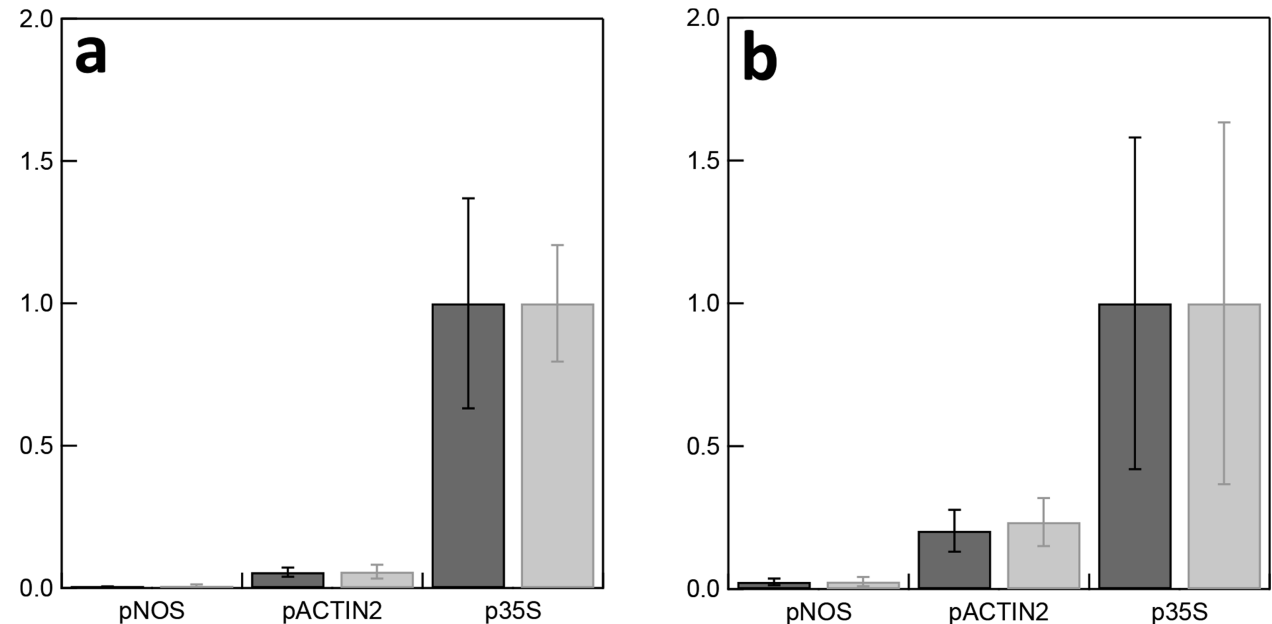

Figure 4. Quantitative assessment of transcriptional activity in leaves of C. sativa (a) and N. benthamiana (b) under dark (dark gray bars) and ambient (light gray bars) light conditions. Statistical data based on 12 and 16 samples for each promoter in (a) and (b), respectively.

promoters, which suggested that those samples were at the noise level.

Taking into account the preceding results, we decided to integrate the fluorescence signals over the whole leaf surface for quantification. More precisely, we analyzed the distribution of (i) the Pre-OPIOM and Speed OPIOM signals from the images collected at $525 \mathrm{~nm}$ with $480 / 405 \mathrm{~nm}$ modulated illumination and (ii) the in-phase DsRed signal from the images collected at $585 \mathrm{~nm}$ with $550 \mathrm{~nm}$ modulated illumination, both acquired on the leaves of $C$. sativa and $N$. benthamiana precedingly observed in the dark.

Figure $3 \mathrm{a}-\mathrm{c}$ displays the results obtained in $C$. sativa seedlings. The in-phase DsRed reporting signal used for plant selection was much lower in the wild type than in the Dronpa2-expressing lines (see Figure 3a). Furthermore, as expected from the DsRed synthesis under the control of the same ubiquitous promoter, it was found to be essentially similar in the three Dronpa-2-expressing lines, albeit with a significant dispersion. The Pre-OPIOM signals were essentially similar and moderately dispersed in all the plants either producing Dronpa-2 or not (see Figure 3b). This result probably originates from the significant plant autofluorescence, which dominates any Dronpa-2-induced Pre-OPIOM signal. In contrast, the Speed OPIOM signals proved discriminating, although with some dispersion (see Figure 3c). First, Speed OPIOM could clearly discriminate the Dronpa-2-expressing lines from the wild-type plants. Moreover, the Speed OPIOM signals were decaying in the expected order of the promoter strengths: $\mathrm{p} 35 \mathrm{~S}>\mathrm{pACTIN} 2>$ pNOS $\gg$ wild type. However, one should notice that the three investigated lines exhibited different levels of Dronpa-2 expression, which could be caused by the positional effect of the construct in the plant genome. We essentially made similar observations on transiently transfected $N$. benthamiana leaves (see Figure S5a-c).

We ended up our analysis by examining whether ambient light would interfere to measure the Dronpa-2 expression level. Based on our preceding results, we directly compared the Dronpa-2 Speed OPIOM signals obtained in the dark and under ambient light acquired on the leaves of $C$. sativa and $N$. benthamiana. In seedlings of $C$. sativa, we observed the expected ranking of expression level, p35S:DRONPA-2 $\gg$ pACTIN2:DRONPA-2 > pNOS:DRONPA-2, the latter construct showing only little trace of expression, which remained below the noise level (see Figure 4a). Moreover, we observed similar levels of expression when the observation was performed in the dark or in ambient light. The analysis of independent transformation events in $N$. benthamiana led to similar conclusions. Overall, no statistical difference was 

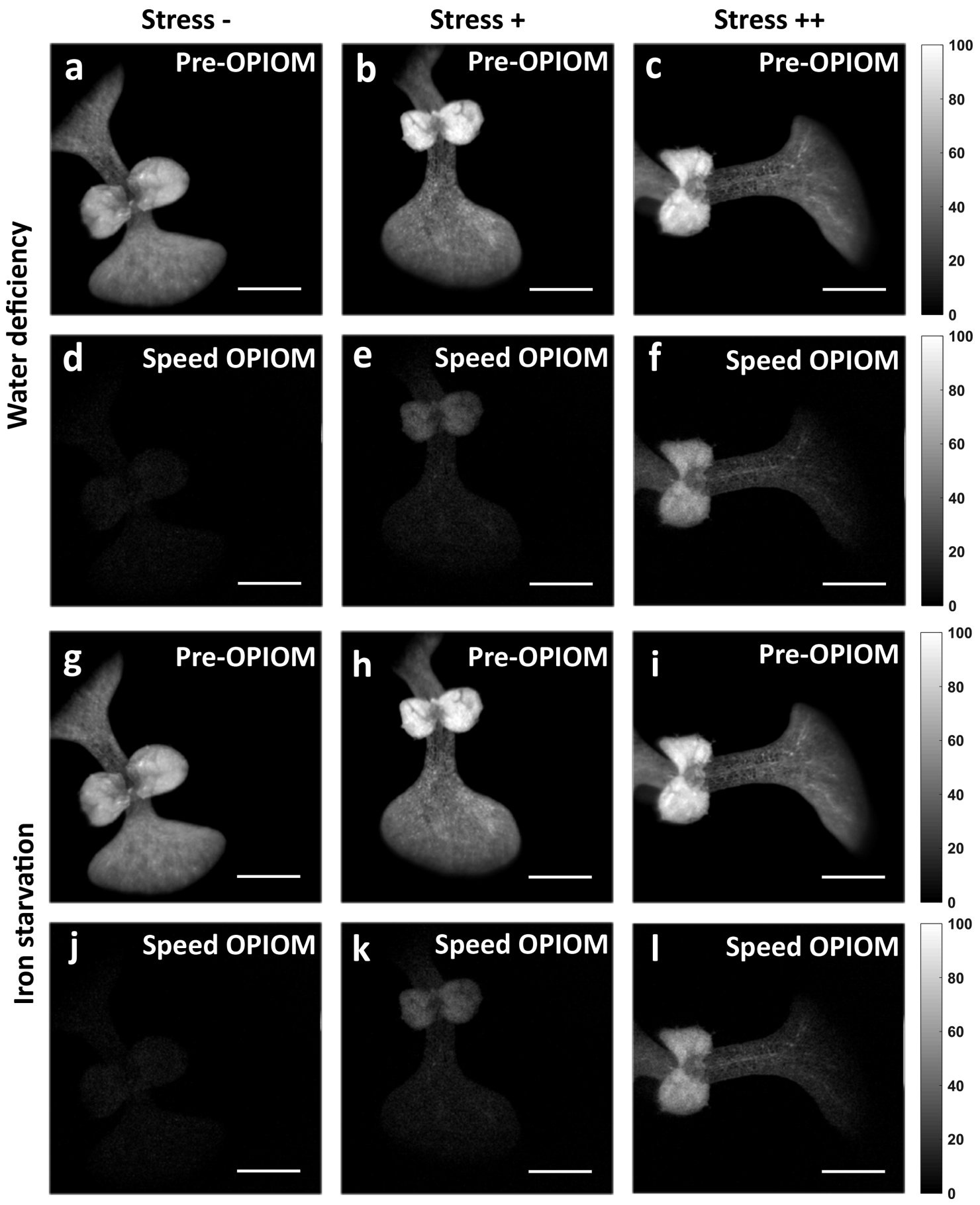

Figure 5. Speed OPIOM for monitoring stresses. ( $a-f)$ Dronpa-2-labeled A. thaliana plants cultivated in soils with different water contents: (a, d) $60 \%$, (b, e) $30 \%$, and (c, f) $20 \%$. The Pre-OPIOM images $(\mathrm{a}-\mathrm{c}$ ) show no visible difference between the three individuals in terms of fluorescence signal. In contrast, the comparison of the Speed OPIOM images $(\mathrm{d}-\mathrm{f})$ reveals the suffering of the plants lacking water; (g-1) Dronpa-2-labeled A. thaliana plants cultivated in soils with different iron availability: (g, j) Fe-abundant, $(h, k)$ trace of Fe mineral, ( $i$, $j$ ) no Fe mineral. The Pre-OPIOM images $(a-c, g-i)$ show a minor difference between the three individuals, whereas the Speed OPIOM images $(d-f, j-l)$ give direct evidence of distinct expression levels of Dronpa-2 without the interference of autofluorescence. Scale bar, $1 \mathrm{~mm}$.

observed between the two sets of data collected in the dark or under ambient light, which indicated that the latter had no influence on quantitative analysis of gene expression by Speed OPIOM (see Figure 4b).

As a last application of Speed OPIOM for quantitative evaluation of gene expression in transgenic plants, we addressed plant monitoring, which is presently demanded for high-thoughput screening in plant selection or for enabling parsimonious cultivation practices optimizing irrigation, fertilization, and pesticide treatment. ${ }^{43}$ Fluorescence is herein advantageous since it is sensitive and compatible with remote monitoring. Hence, endogeneous fluorescence emission from the photosystems of the photosynthetic apparatus provides information on biochemical and anatomical status down to individual plants through the canopy as well as the field and regional scales. ${ }^{44}$ As an alternative possibly endowed with 
improved selectivity, reporter genes driving expression of fluorescent reporters have been suggested for monitoring the plant response to biotic or abiotic stresses. ${ }^{13,16}$ However, this approach is sensitive to environmental light interference and to the plant autofluorescence, which may obscure quantitative monitoring. Since we showed in the preceding subsections that Speed OPIOM was able to evidence and quantify the expression levels of an RSFP, we evaluated the relevance of this imaging protocol for stress detection via reporter genes under ambient light.

We first expressed Dronpa-2 in A. thaliana plants under the control of the VSP2-coding acidic phosphatase, the expression of which is regulated by several biotic and abiotic stresses including water deficiency. ${ }^{45}$ AtNRAMP4 is coding for a vacuolar metal transporter, the expression of which is regulated by iron limitation. ${ }^{46}$ Whereas Pre-OPIOM could not distinguish the plants submitted to three water conditions (Figure 5a-c), Speed OPIOM revealed that soil-drying conditions induced Dronpa-2 expression correlating with the stress level, mainly in petioles of older leaves and cotyledons (Figure 5d-f). Then, we expressed Dronpa-2 in A. thaliana plants under the control of the AtNRAMP4 promoter coding for a vacuolar metal transporter, the expression of which is regulated by iron limitation. Pre-OPIOM was again unable to make the difference between the plants submitted to the three iron conditions (Figure $5 \mathrm{~g}-\mathrm{i}$ ). In contrast, Speed OPIOM could detect Dronpa-2 expression increasing with the stress level, specifically in young leaves of iron-starved plants (Figure $5 j-1)$. Hence, we established the proof of principle that Speed OPIOM was able to counteract autofluorescence and ambient light for reliably evidencing of different levels of expression of an RSFP at specific organs in response to application of stresses of various intensities.

\section{CONCLUDING REMARKS}

This work demonstrates the relevance of Speed OPIOM for remote imaging of RSFPs in the context of plant phenotyping. Speed OPIOM can (i) efficiently distinguish RSFP from autofluorescence and other fluorescent reporters like GFP and DsRed in transient and stable gene expression systems; (ii) quantitatively assess gene expressions, even when they are weak; (iii) be as quantitative, sensitive, and robust in dark and bright light conditions; and (iv) be used to nondestructively record abiotic stress responses like water or iron limitations in real time at the level of individual plants and even of specific organs.

Speed OPIOM validation offers favorable opportunities. Speed OPIOM should be appropriate to monitor gene expression or gene silencing, as well as transposon activity. ${ }^{47,48}$ It could also provide significant information to identify the best plant lines in selection programs by using gene expression as a proxy of an expected phenotype and eventually design predictive markers for specific traits. A third possible use of Speed OPIOM could be to turn plants into environmental sensors. ${ }^{13,16}$ Plants have a large leaf and root exchange surface and also can possibly accumulate metabolites, turning them into sensitive environmentally connected organisms. Speed OPIOM could therefore be used to monitor gene expression associated with pollutants (soil or air), the presence of pathogens, water, or nitrogen content. Eventually, Speed OPIOM could be exploited for assessing gene flow from RSFP-labeled transgenic crops to wild relatives and for ecological monitoring of transgenic plants in the environment, which is an ever important factor in biotechnology risk assessment. $^{49}$

\section{METHODS}

4.1. The Speed OPIOM Macroscope. Our macroscope setup has been previously reported. ${ }^{34}$ Two high-power colored LED chips are used as excitation sources. The blue color LED (LXZ1-PB01, Lumileds) gives a luminance of $L=105-170$ $\mathrm{mW} \cdot \mathrm{mm}^{-2} \cdot \mathrm{sr}^{-1}$ at $500 \mathrm{~mA}$, and the UV LED (LHUV-0400, Lumileds) has a luminance of $L=135-170 \mathrm{~mW} \cdot \mathrm{mm}^{-2} \cdot \mathrm{sr}^{-1}$ at $500 \mathrm{~mA}$. Owing to a condensing system, the two color lights are focused $120 \mathrm{~mm}$ away within an area of $4 \times 4 \mathrm{~mm}^{2}$. Hence, they can deliver an illumination intensity of up to $10 \mathrm{~mW} /$ $\mathrm{mm}^{2}$, with which we are able to reach a modulation frequency of $2.5 \mathrm{~Hz}$.

4.2. The Speed OPIOM Microscope. Our microscopy setup has been previously reported. ${ }^{33}$ We used a home-built epifluorescence microscopy setup equipped with a Luca-R CCD camera (Andor Technology, Belfast, U.K.). Two highpower colored LED chips (LXZ1-PB01 and LHUV-0400, Lumileds) are filtered at $480 \pm 20 \mathrm{~nm}$ (with F480-40; Semrock, Rochester, NY) or at $405 \pm 20 \mathrm{~nm}$ (F405-40; Semrock) and used as excitation sources. The two color lights are collimated with lenses $(f=20 \mathrm{~mm})$, combined with a dichroic mirror (T425LPXR, Chroma, Bellow Falls, VT), and focused at the back focal plane of a $10 \times$ objective $(\mathrm{NA}=0.5$; Carl Zeiss AG, Feldbach, Switzerland) using lenses ( $f=20 \mathrm{~mm}$ and $f=75 \mathrm{~mm}$ ) after being reflected by a dichroic mirror (DiFF506, Semrock, Rochester, NY). Hence, they can deliver an illumination intensity of up to $5 \mathrm{~mW} / \mathrm{mm}^{2}$, with which we are able to reach a modulation frequency of $1 \mathrm{~Hz}$.

4.3. Constructs. 4.3.1. Expression of Dronpa-2, Dronpa3, rsFastlime, and Padron under the Control of the $35 \mathrm{~S}$ Promoter in Tobacco. PCR were performed with primers LG403DRONPAgtwFOR (GGGGACAAGTTTGTACAAAAAAGCAGGCTTCATGGTGAGTGTGATTAAACC) and LG404DRONPAgtwREV (GGGGACCACTTTGTACAAGAAAGCTGGGTCttaCTTGGCCTGCCTCGGCA) to amplify the gateway-modified DRONPA, DRONPA-2 and DRONPA-3, coding sequences using, respectively, the corresponding DNA template. Same procedure was used to amplify the PADRON and rsFastLime gateway-modified coding sequence using, respectively, the LG890PADRONgtwFOR (GGGGACAAGTTTGTACAAAAAAGCAGGCTTCatgagtgtgattaaccaga) and LG891PADRONgtwREV (GGGGACCACTTTGTACAAGAAAGCTGGGTCttagttggcetgcctcggca) primers or LG895rsFastLimegtwFOR (GGGGACAAGTTTGTACAAAAAAGCAGGCTTCatgagtgtgattaaaccaga) or LG896rsFastLimegtwREV (GGGGACCACTTTGTACAAGAAAGCTGGGTCttacttggctgcctcggca) primers. These CDS were then cloned into the pDONR207 plasmid and then transferred into the pB2GW7 vector to obtain the final p35S: fluorescent protein constructs. ${ }^{50}$

4.3.2. Camelina Expression Vector Expressing p35S::Dronpa2 Constructs. The Camelina expression vector expressing p35S::Dronpa 2 construct was achieved as follows. The gateway cassette from pUBN-GFP-Dest $^{51}$ amplified by PCR using primers

- $5^{\prime}$-tgacgcgtaaggggatctagaatcacaagtttgtacaaaaaagctg-3' and

- $5^{\prime}$-ttaatcacactcaccatctcgaggatcaccactttgtacaagaaag-3'. 
and Dronpa-2 coding sequence amplified from Dronpa2pDONR207 with primers

- $5^{\prime}$-atggtgagtgtgattaaaccagaca-3' and

- $5^{\prime}$-ggctgcggccgcctcgactacttggcctgcctcggcagctcag-3'.

were cloned in one step in the BamHI- and XhoI-digested pBinGlyRed vector, ${ }^{52}$ creating $\mathrm{pD} 2$.

4.3.2.1. p35S:DRONPA2; pCMV:DsRED. The doubled CaMV $35 \mathrm{~S}$ promoter was amplified from $\mathrm{pMDC} 83^{53}$ with primers

- $5^{\prime}$-ggggacaagtttgtacaa a a a caggcttccagtgccaagcttggcgtgcct- $3^{\prime}$ and

- 5' -ggggaccactttgtacaagaaagctgggtcgtcgaggtcctctccaaatgaaatg-3

recombined into pDONR207 (Invitrogen) and eventually recombined into $\mathrm{pD} 2$.

4.3.2.2. $p A C T I N 2: D R O N P A 2 ; p C M V: D S R E D$. The actin promoter was amplified from $A$. thaliana Col-0 genomic DNA with primers

- 5'-GGGGACAAGTTTGTACAAAAAAGCAGGCTTCaccatagaatagctagctaactaatac- $3^{\prime}$ and

- 5' - G G G G A C C A C T T T G T A C A A GAAAGCTGGGTCtttttatgagctgcaaacacacaaaaagag-3

recombined into pDONR207 and eventually recombined into $\mathrm{pD} 2$.

4.3.2.3. pNOS:DRONPA2; $p C M V: D s R E D$. The nos promoter was amplified from $\mathrm{pGWB}^{54}$ with primers

- 5'-GGGGACAAGTTTGTACAAAAAAGCAGGCTTCgatcatgagcggagaattaagggag- $3^{\prime}$ and

- 5' - G G G G A C C A C T T T G T A C A A GAAAGCTGGGTCgcgaaacgatccagatccggtgcag-3

recombined into pDONR207 and eventually recombined into $\mathrm{pD} 2$.

4.3.2.4. prVSP2:DRONPA2; $p C M V: D s R E D$. The VSP2 promoter (AT5G24770) was amplified from A. thaliana Col0 genomic DNA with primers

- 5' - G G G G A C A A G T T T G T A C A A A A A A G CAGGCTTCgctgcttggacgattttgtgaatga- $3^{\prime}$ and

- 5' - G G G G A C C A C T T T G T A C A A GAAAGCTGGGTCgttttttatggtatggtttattgtt-3

recombined into pDONR207 and eventually recombined into $\mathrm{pD} 2$.

4.3.2.5. prNRAMP4:DRONPA2; $p C M V: D s R E D$. The AtNRAMP4 promoter (AT5G67330) was amplified from $A$. thaliana Col-0 genomic DNA with primers

- 5' - G G G G A C A A G T T T G T A C A A A A A A G CAGGCTTCttttcttaattgtagtcctaataac- $3^{\prime}$ and

- 5'-GGGGACCACTTTGTACAAGAAAGCTGGGTCatttcccggtaaccgccgattctcag- $3^{\prime}$

recombined into pDONR207 and eventually recombined into pD2.

4.4. Plant Transformation. 4.4.1. Transient Expression in $N$. benthamiana Leaves. The vectors were transformed into Agrobacterium tumefaciens and inoculated in N. benthamiana leaves of 1 month-old plants. Agrobacteria carrying clones of interest were grown overnight at $28{ }^{\circ} \mathrm{C}$ in $5 \mathrm{~mL}$ of $\mathrm{LB}$ medium with appropriate antibiotics. Aliquots from the overnight cultures were resuspended in $10 \mathrm{mM} \mathrm{MgCl}$ and 1 $\mathrm{mM}$ 2-(N-morpholine)-ethanesulfonic acid (MES) to obtain a final $\mathrm{OD}(600 \mathrm{~nm})$ of 0.5 for tobacco leaf infiltration. Two individual leaves of two independent plants were infiltrated.

4.4.2. Permanent Expression in C. sativa. Camelina flower dip transformation was carried out as described previously. ${ }^{55}$

4.4.3. Plant Culture. N. benthamiana and C. sativa were grown on soil in a glass house and imaged directly with Speed OPIOM at about $15 \mathrm{~cm}$ distance. The water stress experiment was carried out in a phenoscope. ${ }^{56}$ The plants were germinated and grown in soil in the glass house for 8 days and then adapted to $60 \%$ soil water content in the phenoscope for 8 days in $8 \mathrm{~h} / 16 \mathrm{~h}$ day/night cycle at $21 / 18{ }^{\circ} \mathrm{C}$ and $65 \%$ humidity. Plants were then cultured for 8 days at 30 or $25 \%$ soil water content before being imaged. For iron stress, plants were cultivated for 12 days in $16 / 8 \mathrm{~h}$ light/dark cycle at $22^{\circ} \mathrm{C}$ in ABIS medium ${ }^{57}$ with $50 \mu \mathrm{M}$ FeHBED (Strem Chemicals) for the control condition (media with $\mathrm{Fe}$ ) or without any iron supplement (trace of iron) or supplemented with $10 \mu \mathrm{M}$ ferrozine (Sigma) to remove any trace of iron.

\section{ASSOCIATED CONTENT}

\section{Supporting Information}

The Supporting Information is available free of charge at https://pubs.acs.org/doi/10.1021/acsomega.0c00957.

Illumination conditions for imaging plant leaves with Speed OPIOM and supplementary figures (PDF)

\section{AUTHOR INFORMATION}

\section{Corresponding Authors}

Ludovic Jullien - PASTEUR, Département de chimie, Ecole normale supérieure, PSL University, SorbonneUniversité, CNRS, 75005 Paris, France; Phone: +33 (0)4432 3333; Email: Ludovic.Jullien@ens.psl.eu

Jean-Denis Faure - Universite Paris-Saclay, INRAE, AgroParisTech, Institut Jean-Pierre Bourgin, F-78000 Versailles, France; Email: Jean-Denis.Faure@inrae.fr, +33 (0)3083 3797

Thomas Le Saux - PASTEUR, Département de chimie, École normale supérieure, PSL University, SorbonneUniversité, CNRS, 75005 Paris, France; 이이이.org/0000-0002-2387-2624; Phone: +33 (0)4432 2406; Email: Thomas.Lesaux@ ens.psl.eu

\section{Authors}

Zsolt Kelemen - Universite Paris-Saclay, INRAE, AgroParisTech, Institut Jean-Pierre Bourgin, F-78000 Versailles, France

Ruikang Zhang - PASTEUR, Département de chimie, Ecole normale supérieure, PSL University, SorbonneUniversité, CNRS, 75005 Paris, France

Lionel Gissot - Université Paris-Saclay, INRAE, AgroParisTech, Institut Jean-Pierre Bourgin, F-78000 Versailles, France

Raja Chouket - PASTEUR, Département de chimie, Ecole normale supérieure, PSL University, SorbonneUniversite, CNRS, 75005 Paris, France

Yannick Bellec - Universite Paris-Saclay, INRAE, AgroParisTech, Institut Jean-Pierre Bourgin, F-78000 Versailles, France

Vincent Croquette - Laboratoire de Physique Statistique, Ecole normale supérieure, PSL Research University, Universite de Paris, Sorbonne Universite, CNRS, 75005 Paris, France; Institut de biologie de l'Ecole normale supérieure (IBENS), Ecole 
normale supérieure, CNRS, INSERM, PSL Research University, 75005 Paris, France

Complete contact information is available at:

https://pubs.acs.org/10.1021/acsomega.0c00957

\section{Author Contributions}

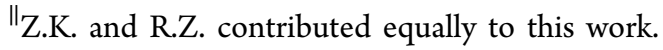

\section{Notes}

The authors declare no competing financial interest.

\section{ACKNOWLEDGMENTS}

The authors thank the ANR (Saclay Plant Sciences (ANR-10LABX-0040-SPS), France BioImaging (ANR-10-INBS-04, ANR-11-IDEX-0003-02), Morphoscope2 (ANR-11-EQPX0029, ANR-17-EUR-0007), EUR SPS-GSR, HIGHLIGHT, and dISCern), the Fondation de la Recherche Médicale (FRM), and the Mission Interdisciplinarite du CNRS. This work has benefited from the support of IJPB's Plant Observatory technological platforms.

\section{REFERENCES}

(1) Gebbers, R.; Adamchuk, V. I. Precision agriculture and food security. Science 2010, 327, 828-831.

(2) Fiorani, F.; Schurr, U. Future scenarios for plant phenotyping. Annu. Rev. Plant Biol. 2013, 64, 267-291.

(3) Chawade, A.; van Ham, J.; Blomquist, H.; Bagge, O.; Alexandersson, E.; Ortiz, R. High throughput field-phenotyping tools for plant breeding and precision agriculture. Agronomy 2019, 9, 258.

(4) Asner, G. P.; Nepstad, D.; Cardinot, G.; Ray, D. Drought stress and carbon uptake in an Amazon forest measured with spaceborne imaging spectroscopy. Proc. Natl. Acad.Sci. U.S.A. 2004, 101, 60396044.

(5) Blackburn, G. A. Hyperspectral remote sensing of plant pigments. J. Exp. Bot. 2006, 58, 855-867.

(6) Baret, F.; Houles, V.; Guerif, M. Quantification of plant stress using remote sensing observations and crop models: the case of nitrogen management. J. Exp. Bot. 2007, 58, 869-880.

(7) Omasa, K.; Hosoi, F.; Konishi, A. 3D lidar imaging for detecting and understanding plant responses and canopy structure. J. Exp. Bot. 2006, 58, 881-898.

(8) Baker, N. R. Chlorophyll fluorescence: a probe of photosynthesis in vivo. Annu. Rev. Plant Biol. 2008, 59, 89-113.

(9) Nedbal, L.; Soukupová, J.; Kaftan, D.; Whitmarsh, J.; Trtílek, M. Kinetic imaging of chlorophyll fluorescence using modulated light. Photosynth. Res. 2000, 66, 3-12.

(10) Kaiser, E.; Morales, A.; Harbinson, J.; Kromdijk, J.; Heuvelink, E.; Marcelis, L. F. M. Dynamic photosynthesis in different environmental conditions. J. Exp. Bot. 2014, 66, 2415-2426.

(11) Dedecker, P.; De Schryver, F. C.; Hofkens, J. Fluorescent proteins: Shine on, you crazy diamond. J. Am. Chem. Soc. 2013, 135, 2387-2402.

(12) Krizek, B. A.; Prost, V.; Joshi, R. M.; Stoming, T.; Glenn, T. C. Developing transgenic arabidopsis plants to be metal-specific bioindicators. Environ. Toxicol. Chem. 2003, 22, 175-181.

(13) Kooshki, M.; Mentewab, A.; Stewart, C. N., Jr. Pathogen inducible reporting in transgenic tobacco using a GFP construct. Plant Sci. 2003, 165, 213-219.

(14) Perera, M. R.; Jones, M. G. Expression of the peroxidase gene promoter (Shpx6b) from Stylosanthes humilis in transgenic plants during insect attack. Entomol. Exp. Appl. 2004, 111, 165-171.

(15) Paul, A.-L.; Schuerger, A. C.; Popp, M. P.; Richards, J. T.; Manak, M. S.; Ferl, R. J. Hypobaric biology: Arabidopsis gene expression at low atmospheric pressure. Plant Physiol. 2004, 134, 215-223.
(16) Liew, O.; Chong, P.; Li, B.; Asundi, A. Signature optical cues: emerging technologies for monitoring plant health. Sensors 2008, 8, 3205-3239.

(17) Norikane, J. H.; Kurata, K. Water stress detection by monitoring fluorescence of plants under ambient light. Trans. ASAE 2001, 44, 1915.

(18) Lanni, F.; Pane, D. A.; Weinstein, S. J.; Waggoner, A. S. Compact flashlamp-based fluorescence imager for use under ambientlight conditions. Rev. Sci. Instr. 2007, 78, No. 033702.

(19) Egly, D.; Geörg, D.; Rädle, M.; Beuermann, T. A compact multi-channel fluorescence sensor with ambient light suppression. Meas. Sci. Technol. 2012, 23, No. 035702.

(20) Sexton, K.; Davis, S. C.; McClatchy, D.; Valdes, P. A.; Kanick, S. C.; Paulsen, K. D.; Roberts, D. W.; Pogue, B. W. Pulsed-light imaging for fluorescence guided surgery under normal room lighting. Opt. Lett. 2013, 38, 3249-3252.

(21) Sexton, K. J.; Zhao, Y.; Davis, S. C.; Jiang, S.; Pogue, B. W. Optimization of fluorescent imaging in the operating room through pulsed acquisition and gating to ambient background cycling. Biomed. Opt. Express 2017, 8, 2635-2648.

(22) Zhu, B.; Rasmussen, J. C.; Sevick-Muraca, E. M. Non-invasive fluorescence imaging under ambient light conditions using a modulated ICCD and laser diode. Biomed. Opt. Express 2014, 5, $562-572$.

(23) Belkin, S.; Yagur-Kroll, S.; Kabessa, Y.; Korouma, V.; Septon, T.; Anati, Y.; Zohar-Perez, C.; Rabinovitz, Z.; Nussinovitch, A.; Agranat, A. J. Remote detection of buried landmines using a bacterial sensor. Nat. Biotechnol. 2017, 35, 308-310.

(24) Zimmermann, T.; Rietdorf, J.; Pepperkok, R. Spectral imaging and its applications in live cell microscopy. FEBS Lett. 2003, 546, 8792.

(25) Fereidouni, F.; Bader, A. N.; Gerritsen, H. C. Spectral phasor analysis allows rapid and reliable unmixing of fluorescence microscopy spectral images. Opt. Express 2012, 20, 12729-12741.

(26) Billinton, N.; Knight, A. W. Seeing the wood through the trees: a review of techniques for distinguishing green fluorescent protein from endogenous autofluorescence. Anal. Biochem. 2001, 291, 175197.

(27) Quérard, J.; Le Saux, T.; Gautier, A.; Alcor, D.; Croquette, V.; Lemarchand, A.; Gosse, C.; Jullien, L. Kinetics of reactive modules adds discriminative dimensions for selective cell imaging. ChemPhysChem 2016, 17, 1396-1413.

(28) Bastiaens, P. I.; Squire, A. Fluorescence lifetime imaging microscopy: spatial resolution of biochemical processes in the cell. Trends Cell Biol. 1999, 9, 48-52.

(29) Kodama, Y. Time gating of chloroplast autofluorescence allows clearer fluorescence imaging in planta. PLoS One 2016, 11, e0152484.

(30) Bourgeois, D.; Adam, V. Reversible photoswitching in fluorescent proteins: a mechanistic view. IUBMB Life 2012, 64, 482-491.

(31) Zhou, X. X.; Lin, M. Z. Photoswitchable fluorescent proteins: ten years of colorful chemistry and exciting applications. Curr. Opin. Chem. Biol. 2013, 17, 682-690.

(32) Lummer, M.; Humpert, F.; Wiedenlübbert, M.; Sauer, M.; Schüttpelz, M.; Staiger, D. A new set of reversibly photoswitchable fluorescent proteins for use in transgenic plants. Mol. Plant 2013, 6, $1518-1530$

(33) Quérard, J.; et al. Resonant out-of-phase fluorescence microscopy and remote imaging overcome spectral limitations. Nat. Commun. 2017, 969.

(34) Zhang, R.; Chouket, R.; Plamont, M.-A.; Kelemen, Z.; Espagne, A.; Tebo, A. G.; Gautier, A.; Gissot, L.; Faure, J.-D.; Jullien, L.; Croquette, V.; Le Saux, T. Macroscale fluorescence imaging against autofluorescence under ambient light. Light Sci. Appl. 2018, 97.

(35) Querard, J.; Markus, T.-Z.; Plamont, M.-A.; Gauron, C.; Wang, P.; Espagne, A.; Volovitch, M.; Vriz, S.; Croquette, V.; Gautier, A.; Le Saux, T.; Jullien, L. Photoswitching Kinetics and Phase-Sensitive Detection Add Discriminative Dimensions for Selective Fluorescence Imaging. Angew. Chem. Int. Ed. 2015, 54, 2633-2637. 
(36) Liang, H.; Kumar, P. A.; Nain, V.; Powell, W. A.; Carlson, J. E. Transgenic Crop Plants; Kole, C; Michler, C. H., Abbott, A. G., Hall, T. C., Eds.; Springer: Berlin Heidelberg, 2010; pp 85-143.

(37) Stiel, A. C.; Trowitzsch, S.; Weber, G.; Andresen, M.; Eggeling, C.; Hell, S. W.; Jakobs, S.; Wahl, M. C. 1.8 Å bright-state structure of the reversibly switchable fluorescent protein Dronpa guides the generation of fast switching variants. Biochem. J. 2007, 402, 35-42.

(38) Ando, R.; Mizuno, H.; Miyawaki, A. Regulated fast nucleocytoplasmic shuttling observed by reversible protein highlighting. Science 2004, 306, 1370-1373.

(39) Martin, K.; Kopperud, K.; Chakrabarty, R.; Banerjee, R.; Brooks, R.; Goodin, M. M. Transient expression in Nicotiana benthamiana fluorescent marker lines provides enhanced definition of protein localization, movement and interactions in planta. Plant $J$. 2009, 59, 150-162.

(40) Sarrion-Perdigones, A.; Vazquez-Vilar, M.; Palací, J.; Castelijns, B.; Forment, J.; Ziarsolo, P.; Blanca, J.; Granell, A.; Orzaez, D. GoldenBraid 2.0: a comprehensive DNA assembly framework for plant synthetic biology. Plant Physiol. 2013, 162, 1618-1631.

(41) Halfhill, M.; Millwood, R.; Rufty, T.; Weissinger, A.; Stewart, C. N., Jr. Spatial and temporal patterns of green fluorescent protein (GFP) fluorescence during leaf canopy development in transgenic oilseed rape, Brassica napus L. Plant Cell Rep. 2003, 22, 338-343.

(42) Mazarei, M.; Teplova, I.; Hajimorad, M. R.; Stewart, C. N., Jr. Pathogen phytosensing: plants to report plant pathogens. Sensors 2008, 8, 2628-2641.

(43) Grierson, C. S.; Barnes, S. R.; Chase, M. W.; Clarke, M.; Grierson, D.; Edwards, K. J.; Jellis, G. J.; Jones, J. D.; Knapp, S.; Oldroyd, G.; et al. One hundred important questions facing plant science research. New Phytol. 2011, 192, 6-12.

(44) Meroni, M.; Rossini, M.; Guanter, L.; Alonso, L.; Rascher, U.; Colombo, R.; Moreno, J. Remote sensing of solar-induced chlorophyll fluorescence: Review of methods and applications. Remote Sens. Environ. 2009, 113, 2037-2051.

(45) Huang, D.; Wu, W.; Abrams, S. R.; Cutler, A. J. The relationship of drought-related gene expression in Arabidopsis thaliana to hormonal and environmental factors. J. Exp. Bot. 2008, 59, 29913007.

(46) Lanquar, V.; Leliévre, F.; Bolte, S.; Hamés, C.; Alcon, C.; Neumann, D.; Vansuyt, G.; Curie, C.; Schröder, A.; Krämer, U.; Barbier-Brygoo, H.; Thomine, S. Mobilization of vacuolar iron by AtNRAMP3 and AtNRAMP4 is essential for seed germination on low iron. EMBO J. 2005, 24, 4041-4051.

(47) Stewart, C. N., Jr. Monitoring the presence and expression of transgenes in living plants. Trends Plant Sci. 2005, 10, 390-396.

(48) Rosellini, D. Selectable markers and reporter genes: a well furnished toolbox for plant science and genetic engineering. Crit. Rev. Plant Sci. 2012, 31, 401-453.

(49) Wei, W.; Kwit, C.; Millwood, R. J.; Moon, H. S.; Stewart, C. N., Jr. In Plant Gene Containment; Melvin, J. O., Yi, L., Eds.; John Wiley \& Sons, Ltd, 2012; Vol. 27; Chapter Assessment and detection of gene flow, $\mathrm{p} 37$.

(50) Karimi, M.; Inzé, D.; Depicker, A. GATEWAY ${ }^{\mathrm{TM}}$ vectors for Agrobacterium-mediated plant transformation. Trends Plant Sci. 2002, 7, 193-195.

(51) Grefen, C.; Donald, N.; Hashimoto, K.; Kudla, J.; Schumacher, K.; Blatt, M. R. A ubiquitin-10 promoter-based vector set for fluorescent protein tagging facilitates temporal stability and native protein distribution in transient and stable expression studies. Plant $J$. 2010, 64, 355-365.

(52) Nguyen, H. T.; Silva, J. E.; Podicheti, R.; Macrander, J.; Yang, W.; Nazarenus, T. J.; Nam, J.-W.; Jaworski, J. G.; Lu, C.; Scheffler, B. E.; Mockaitis, K.; Cahoon, E. B. Camelina seed transcriptome: a tool for meal and oil improvement and translational research. Plant Biotechnol. J. 2013, 11, 759-769.

(53) Curtis, M. D.; Grossniklaus, U. A gateway cloning vector set for high-throughput functional analysis of genes in planta. Plant Physiol. 2003, 133, 462-469.
(54) Nakagawa, T.; Kurose, T.; Hino, T.; Tanaka, K.; Kawamukai, M.; Niwa, Y.; Toyooka, K.; Matsuoka, K.; Jinbo, T.; Kimura, T. Development of series of gateway binary vectors, pGWBs, for realizing efficient construction of fusion genes for plant transformation. $J$. Biosci. Bioeng. 2007, 104, 34-41.

(55) Morineau, C.; Bellec, Y.; Tellier, F.; Gissot, L.; Kelemen, Z.; Nogué, F.; Faure, J.-D. Selective gene dosage by CRISPR-Cas9 genome editing in hexaploid Camelina sativa. Plant Biotechnol. J. 2017, 15, 729-739.

(56) Tisné, S.; Serrand, Y.; Bach, L.; Gilbault, E.; Ben Ameur, R.; Balasse, H.; Voisin, R.; Bouchez, D.; Durand-Tardif, M.; Guerche, P.; Chareyron, G.; Da Rugna, J.; Camilleri, C.; Loudet, O. Phenoscope: an automated large-scale phenotyping platform offering high spatial homogeneity. Plant J. 2013, 74, 534-544.

(57) Oomen, R. J. F. J.; Wu, J.; Leliévre, F.; Blanchet, S.; Richaud, P.; Barbier-Brygoo, H.; Aarts, M. G.; Thomine, S. Functional characterization of NRAMP3 and NRAMP4 from the metal hyperaccumulator Thlaspi caerulescens. New Phytol. 2009, 181, 637-650. 